\title{
The Invention Coach: A computer-based environment that supports the transfer of STEM concepts
}

\author{
Marianna Lamnina, ${ }^{1}$ Helena Connolly, ${ }^{1}$ Vincent Aleven, ${ }^{2}$ and Catherine C. Chase ${ }^{1}$ \\ ${ }^{1}$ Human Development, Teachers College, Columbia University, 525 W $120^{\text {th }}$ St., New York, NY 10027 \\ ${ }^{2}$ Human Computer Interaction Institute, Carnegie Mellon University, 5000 Forbes Ave., Pittsburgh, PA, 15213
}

\begin{abstract}
This work explores ways to scaffold invention activities to facilitate productive exploration of ratio structures in physical science equations, the goal of which is to prepare students to learn from later expository instruction. We have developed the first computerized Invention Coach that provides adaptive guidance as middle school students work through invention tasks. This paper will discuss the rationale behind our novel pedagogical model, which draws upon empirical studies of human teachers guiding invention, as well as prior research on the core learning processes that invention promotes. Preliminary findings from a classroom study of the Invention Coach provide some evidence of the system's efficacy in enhancing transfer.
\end{abstract}

\section{INTRODUCTION}

Invention is an instructional technique in which learners solve ill-structured problems by inventing their own solutions, methods, or equations. One effective form of invention involves contrasting cases. Contrasting cases are sets of examples that vary on deep features of the domain. These materials are designed to help students notice information they might otherwise overlook, such as the roles certain variables play. Contrasting cases are particularly effective when students invent solutions prior to receiving direct instruction of the concept [1]. This pedagogy serves as preparation for future learning. By engaging in invention activities and grappling with concepts before they are taught them directly, students are better prepared to learn from later instruction [1]. Invention prior to direct instruction has strong positive effects on learning transfer, often in science and math domains [2].

An example invention activity is shown in Fig. 1. This example is one of the two activities used in the Invention Coach, and will be used throughout this paper to illustrate aspects of the Coach's design. The activity consists of a set of six contrasting cases of busses packed with clowns. Using these cases, students are to invent an index that describes the crowdedness of each bus. The cases vary systematically in size of the bus and number of clowns, so that students could make contrasts that hold one of these features constant, while varying the other. They are given

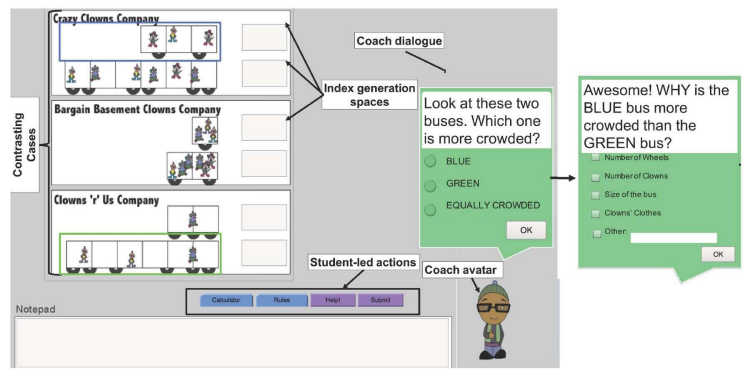

FIG. 1. Invention Coach main interface as a student progresses through the feature contrast module. some rules: they have to use the same method to find the index for each bus, busses from the same bus company have the same crowdedness, and the more crowded the bus, the higher the index. Grappling with this problem is meant to prepare students to learn about density and other ratiobased physical science equations, from direct instruction.

However, to productively prepare students to take advantage of direct instruction, invention requires subtle individualized expert guidance [3]. In fact, classroom studies of invention typically involve one teacher for every five students [4]. This can make invention impractical in everyday classrooms. Therefore, we sought to design a computerized, adaptive Invention Coach that would provide optimal guidance as students muddle through the often murky and messy process of invention. A study of human teacher guidance of invention, along with prior research and theory, provided inspiration for the Coach's general style of feedback. In this paper, we describe our design process and report on a study evaluating the effectiveness of the Invention Coach software.

\section{DESIGNING THE INVENTION COACH}

Our goal in designing the Invention Coach was to create an adaptive, naturalistic system to support students through the invention process. To do this, we conducted a study of human teachers guiding students through invention. From this study, we identified a general style of guidance and specific forms of feedback that facilitated or hindered learning transfer. These findings informed the design of our Invention Coach, which employed an "ask more, tell less" style of feedback. Next, from prior research and theory, we drew on three cognitive processes of invention that lead to transfer, and designed an interactive module to support each of these processes.

\section{A. Study of Human Teacher Guidance}

Computer-based guidance can feel forced and unnatural. We wanted our system to have a naturalistic, human feel, based on a model of human teacher guidance. Therefore, we embarked on a study of experienced science teachers 
guiding students one-on-one through invention activities [3].

We asked teachers not to tell students the answers during the invention activities. Otherwise, they guided students naturally. Gains from pretest to posttest showed that the teachers were quite successful in increasing students' conceptual knowledge (effect size $d=0.6$ ) and ability to transfer to novel domains $(d=0.7)$. Overall, we found that teacher-student dialogue was very studentcentered. Teachers asked questions twice as often as they gave explanations, and they rarely gave direct feedback. Moreover, we found that the more often teachers explained, the less students were able to transfer what they learned from the invention activity to novel problems.

Given these findings, we designed the Invention Coach with an "ask more, tell less" feedback style. Instead of giving didactic explanations, the Coach prompts students to reflect on and explain their answers. Instead of stating the errors and how to fix them, the Coach problematizes students' work [6]. When problematizing, the Coach contradicts and pokes holes in incorrect solutions, and encourages students to diagnose their own errors. This is different from other Intelligent Tutoring Systems, which tend to be fairly teacher-centered, and provide direct, corrective, and/or explanatory feedback [7].

\section{B. Prior Research and Theory}

In addition to our work with human teachers, we also drew on prior research and theory on the cognitive processes that make invention effective. These mechanisms are thought to be (1) activation of prior knowledge, (2) realization of knowledge gaps, and (3) noticing of deep features of the problem. Based on these three mechanisms, we designed three modules, each addressing a mechanism. Each module involved a brief interactive activity in the form of a set of prompts from the Coach avatar for the student to respond to within the system.

\section{Activating Prior Knowledge: The Ranking Module}

Activation of prior knowledge is key in productive invention activities. When inventing, students use their prior knowledge to invent solutions to an unfamiliar, illstructured problem [8]. Therefore, invention activities will be most effective when they include at least some features with which students are already familiar. In the "clown crowdedness" activity described earlier, students were inventing density, but through an artificial example of clowns packed in busses. While students might not have had experience with solving for density and almost certainly did not have experience in coming up with a number to explain how crowded a bus full of clowns is, they most likely understood intuitively the difference between more and less crowded busses.

To support this intuitive understanding, we developed a ranking module. The ranking module prompts students to rank the cases from most crowded to least crowded. Doing so leads students to bring in their prior knowledge of crowdedness, which often provides students who are lost with a way to get started on the problem. For other students, ranking drives them to compare their index numbers to their intuitive understanding of crowdedness. For instance, if a student dragged the buses into the correct order, but entered the wrong indices, $\mathrm{s} /$ he might notice that the index numbers were out of order. Thus, by engaging prior knowledge, $\mathrm{s} / \mathrm{he}$ is also noticing her/his current knowledge gaps, a second mechanism for the effectiveness of invention.

\section{Noticing Knowledge Gaps: The Tell-Me-How Module}

Noticing gaps in current knowledge is essential during the invention process because when students notice these gaps, they can understand canonical solutions more deeply (i.e. take advantage of direct instruction later) [9]. These gaps are more likely to be noticed when they are experienced than when they are told [10]. Therefore, invention is an opportune activity for students to notice their knowledge gaps because they will likely do so when they see that their inventions do not match the constraints of the problem.

For example, in the clown crowdedness activity, students were given sets of contrasting cases (e.g. buses packed with clowns) and were given some constraints to work within (e.g. "Use the same method to find each index."). Noticing that their solution violated one of these constraints would naturally lead students to notice a gap in their understanding of the problem. To facilitate this, we developed a tell-me-how module that prompted students to self-explain their solutions. In this module, the Coach asks the student to explain how s/he came up with each index number. Once the student explains her/his method, a small box with the method appears next to the appropriate case (e.g. the appropriate bus). This can lead students to notice gaps in his/her knowledge by making explicit their inconsistencies or violations of constraints. For instance, because the methods the student enters appear on the screen next to each case, if a student entered the same method for two cases (e.g. clowns divided by spaces), but a different method for another one (e.g. counting clowns), this inconsistency is now displayed clearly on the screen, leading the student to notice a gap in his understanding. Moreover, once students notice their gaps, they may begin to search for new solutions or methods, and in the process, notice the deep features of the problem, the third mechanism of productive invention.

\section{Noticing Deep Features: The Feature Contrast Module}

Noticing deep features is characteristic of inventing with contrasting cases, since the cases systematically vary in these features. When students notice these features, it becomes easier to transfer them to novel situations [10]. 
However, when students are not told explicitly to examine the contrasting cases, they tend to focus on irrelevant features, whereas when they are, they spontaneously explain the deep features three times as much [11]. Therefore, we developed the feature contrast module (see Fig. 1) to make use of the contrasting cases and guide students in noticing the deep features of the problem.

This module selects two cases that vary on a single critical feature (e.g. holds number of clowns constant, while varying bus size) and asks the student first, to compare them (i.e. "Which bus is more crowded?") and then, to explain their comparison (i.e. "Why?"). Next, the Coach selects two cases that contrast on a different critical feature (e.g. holds bus size constant, but varies the number of clowns), and repeats the questions. Finally, it prompts the student to put together what s/he learned from the two comparisons into a singular explanation, thereby guiding her/him to relate the deep features of the problem.

\section{EFFICACY OF DIFFERENT AMOUNTS OF COACH GUIDANCE}

Because it can be difficult to know the optimal amount of feedback to give in ill-structured problem-solving tasks, we compared the full version of the Invention Coach to a minimally guided version and an unguided version of the software. This study also served as an initial study of the Coach's efficacy; if our designed guidance is effective, the full guidance version should lead to the greatest transfer.

In all three conditions, students engaged in an iterative process of inventing and re-inventing their index numbers. Throughout this process, students had access to studentinitiated tools such as a calculator, a notepad, and an introduction to the problem. Each time students "submit" their indices, they received some form of feedback, which varied by condition. In the no guidance version, the only feedback students received was a suggestion to keep working as they hadn't quite gotten it yet. The minimally guided version received the same feedback plus feedback about whether their solution met the goals and constraints of the problem (e.g. "Remember, you have to use the EXACT SAME method to find the index for all of the buses"). Finally, in the full guidance version, the feedback consisted of feedback to keep going, goals-and-constraints feedback, plus the three modules explained above. All modules and goals-and-constraints feedback in the full and minimally guided conditions were adaptive, meaning that feedback was selectively chosen to respond to a student's particular type of solution. All three versions also included motivational messages (e.g. "I can see the gears turning in your brain, you're working hard!").

\section{A. Invention Activities}

Students worked on two invention activities: "clown crowdedness," and "car fastness" by inventing an index number to represent these concepts. The clown crowdedness activity, described in the introduction, is meant to prepare students to learn about density. The car fastness activity is meant to prepare students to learn about speed. In the car fastness activity, students were given six contrasting cases of cars, varying systematically in relevant features: distance traveled and time taken (represented with oil drops that drip once per minute).

\section{B. Participants, Methods, and Study Design}

Students from nine seventh- and eighth-grade classes (N $=205$ ) in a public middle school in New Jersey participated in this study over a total of five days during their regular science classes. The school population is $96 \%$ Hispanic and $56 \%$ male. Condition was randomized at the student level, so students within the same class received different versions of the software (full guidance, minimal guidance, no guidance). On the first day, two weeks before the main intervention, they took a pretest. On the second and third days, they worked with the Invention Coach on two activities (clown crowdedness and car fastness). On the fourth day, all students received the same lecture that explained the canonical solution to activities they worked on, how these activities relate to science concepts (density and speed), and arguably most important for transfer, how these concepts rely on a ratio structure, which can be applied to many other scientific equations. Finally, on the fifth day, they completed a posttest.

The pretest and posttest included conceptual items, which required understanding of relationships among the variables in real-world contexts (e.g. determining whether a large or small pillow is more "tightly packed" if the number of feathers is constant). Additionally, the posttest asked application items that asked students about crowdedness/ speed in novel situations (e.g. the crowdedness of flowers in a pot). The posttest also asked transfer questions, testing new science concepts that were not explicitly taught, but relied on the same ratio structure as density and speed (e.g. coming up with a way to describe the "stiffness" [i.e. spring constant] of several trampolines, a ratio of force and displacement).

\section{Results}

First, we wanted to investigate how the conditions affected performance on the invention activities themselves. We found that $49 \%$ of students in the full guidance condition, $59 \%$ of students in the minimal guidance condition, and $65 \%$ of students in the no guidance condition invented a ratio solution on either of the invention activities. This difference was not significant, $\chi^{2}(2)=3.64$, $p=.16$. This is a counterintuitive result, because we had expected the full guidance condition to be most successful on the invention tasks, but this was not so.

Next, to analyze how condition affected posttest measures, we conducted a MANCOVA, with condition as the independent variable, the three posttest measures 
(conceptual understanding, application, and transfer) as dependent variables, and class, pretest score, and whether students invented a ratio solution in either task as covariates. All covariates had significant main effects on the dependent variables ( $p$ 's $<.002$ ). They did not have any significant interactions with any of the variables, so interactions were excluded from our MANCOVA model. The omnibus MANCOVA revealed a significant main effect of condition, $F(6,380)=2.50, p=.022, \eta_{p}{ }^{2}=.04$. Follow-up ANOVAs revealed that all three groups performed similarly on conceptual and application items, $p$ 's $>.141$, but differed significantly on transfer items, $F(2$, $192)=5.04, p=.007$. Posthoc tests revealed that on these transfer items, students in the full guidance condition performed significantly better than those in the no guidance condition, $p=.002$. The minimal guidance condition did not differ significantly from either condition. So by inference, the full guidance condition performed the best, followed by the minimal guidance condition, and then the no guidance condition, which showed the lowest performance (see Fig. 2).

To compare the efficacy of our full Invention Coach to the human tutors in our previous study, we can explore gains on items that were common pre to post. While there were no significant gains on conceptual items, the full guidance group made significant gains on the common transfer item, effect size $d=0.6$. Thus, the full guidance provided by the Invention Coach was almost as effective as

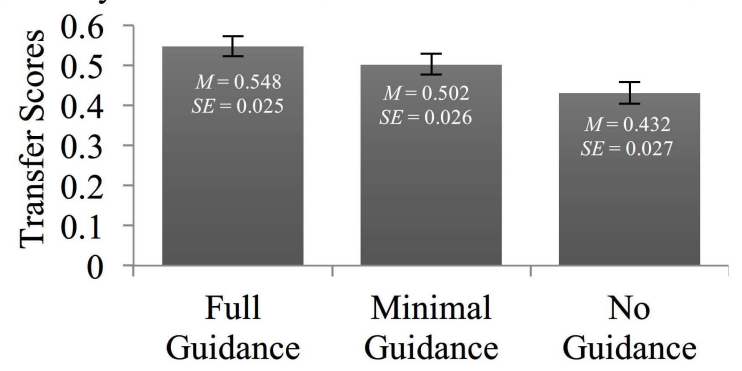

FIG. 2. Adjusted mean transfer scores and standard errors by condition. Max transfer score $=1$. the human tutors used in our previous study in enhancing transfer $(d=0.7)[3]$, but not conceptual learning $(d=0.6)$.

\section{DISCUSSION AND CONCLUSION}

In sum, we have detailed the design of the Invention Coach, which was based on a study of human teacher guidance of invention along with prior research and theory on the cognitive processes that make invention effective at enhancing transfer. Our full guidance version of the Coach has an adaptive, "ask more, tell less" inquiry style of guidance that problematizes student solutions. It also contains activities (modules) that encourage students to activate prior knowledge, identify gaps in their understanding, and notice deep features of the problem.

This work provides initial evidence of the full Invention Coach's efficacy in enhancing transfer. Our study showed that the full guidance version was most effective at inducing transfer, compared to minimal or no guidance versions of the same system. Moreover, the full guidance version was comparable to human teachers in inducing transfer. This research also provides evidence for the optimal level and type of feedback in guiding students through invention activities, when the goal is transfer. Future research should test the efficacy of problematizing feedback specifically (compared to other forms of feedback) and explore ways to enhance conceptual learning from the Invention Coach system.

Finally, this work offers a blueprint for designing software to guide ill-structured problem-solving. We plan to extend the Invention Coach software to guide a broader variety of invention activities and math/science concepts.

\section{ACKNOWLEDGEMENTS}

The authors would like to thank Octav Popescu and Jenna Marks for their instrumental roles in developing the Invention Coach. This work was supported by the National Science Foundation under Grant No. 1361062.
[1] D.L. Schwartz and T. Martin, Cognition and Instruction, 22, 2, (2004).

[2] D.L. Schwartz and J.D. Bransford, Cognition and Instruction, 16, 4, (1998).

[3] C.C. Chase et al., in Proceedings of the Workshops at the 17th AIED International Conference on Artificial Intelligence in Education, 2015, edited by J. Boticario and K. Muldner, p. 1.

[4] J. Marks, D. Bernett, and C.C. Chase, International Journal of Designs for Learning, 7, 2, (2016).

[5] K. Loibl, I. Roll, and N. Rummel, Educational Psychology Review, (2016).
[6] B.J. Reiser, Journal of the Learning Sciences, 13, 3 (2004).

[7] B. du Boulay and R. Luckin, International Journal of Artificial Intelligence in Education, 12, 3 (2001).

[8] M. Kapur, and K. Bielaczyc, The Journal of the Learning Sciences, 21, 1, (2012).

[9]K. VanLehn et al., Cognition and Instruction, 21, 3, (2003).

[10]K. W. Eva, and G. Regehr, Advances in Health Sciences Education, 16, 3 (2011).

[11]I. Roll, N.G. Holmes, J. Day and D. Bonn, Instructional Science, 40, 4, (2012). 